\title{
EFEITO DO ESTRESSE TÉRMICO SOBRE A FREQUÊNCIA CARDÍACA, GASTO ENERGÉTICO, PERDA HÍDRICA E INGESTÃO DE ÁGUA EM JOGADORES DE VOLEIBOL
}

\author{
Alan Pablo Grala ${ }^{1}$ \\ Roni Peterson da Silva ${ }^{2}$ \\ Marcos Antonio Rolim Teixeira ${ }^{3}$ \\ Jefferson Bagarolo de Souza ${ }^{4}$ \\ Bruna dos Santos Vargas ${ }^{5}$ \\ Michel Augusto da Silva ${ }^{6}$
}

GRALA, A. P.; SILVA, R. P. da; TEIXEIRA, M. A. R.; SOUZA, J. B. de; VARGAS, B. dos S.; SILVA, M. A. da. Efeito do estresse térmico sobre a frequência cardíaca, gasto energético, perda hídrica e ingestão de água em jogadores de voleibol. Arq. Ciênc. Saúde Unipar, Umuarama, 19, n. 3, p. 199-203, set./dez. 2015.

RESUMO: A presente pesquisa buscou investigar o efeito de diferentes situações de estresse térmico (WBGT) sobre a frequência cardíaca (FC), gasto energético (GE), perda hídrica (PH) e consumo de água (CA) em jovens atletas de voleibol. Participaram do estudo

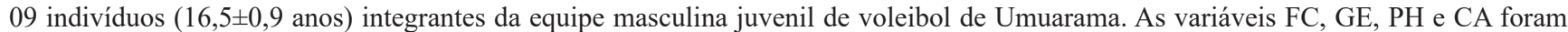
mensuradas durante duas sessões de treinamento $(\mathrm{S} 1, \mathrm{~S} 2)$ de volume $(85 \mathrm{~min})$ e intensidade semelhantes, porém, com diferentes situações de estresse térmico (S1: WBGT:22,62 ${ }^{\circ} \mathrm{C}$; S2: WBTG: $26.55^{\circ} \mathrm{C}$ ). O estresse térmico (WBGT) do ambiente foi determinado por um termômetro de globo modelo ITWTG2000. A FC e o GE foram mensurados por meio do equipamento Sunnto Team Pod. A PH foi calculada pela diferença entre o peso corporal no início e final das sessões de treinamento. O CA foi "ad libitum”, porém, o volume total ingerido foi calculado usando copos descartáveis de $180 \mathrm{ml}$. Para análise dos dados foi utilizado o teste T pareado (amostras dependentes). O índice de significância adotado foi de $\mathrm{p}<0,05$. Após análise dos dados, observou-se similar FC (S1: 135,5 $\pm 16,3 \mathrm{bpm}$ vs. S2: 141,5 $\pm 21,5 \mathrm{bpm}$ ), GE (S1 :994,44 $\pm 267,4 \mathrm{Kcal}$ vs. S2: $881,2 \pm 259,6 \mathrm{Kcal})$, PH (S1: $0,6 \pm 0,2 \mathrm{Kg}$ vs. S2: $0,4 \pm 0,3 \mathrm{Kg}$ ) e CA (S1 :1120 $\pm 345 \mathrm{~mL}$ vs. S2: $760 \pm 429 \mathrm{~mL}$ ) nas duas sessões de treinamento. Os resultados indicam que uma pequena variação no estresse térmico do ambiente não afeta de forma marcante a FC, GE, PH e CA durante o treinamento de voleibol.

PALAVRAS-CHAVE: Estresse térmico; Voleibol; Frequência cardíaca.

\section{THE EFFECT OF THERMAL STRESS ON HEART RATE, ENERGY EXPENDITURE, WATER LOSS AND WATER INTAKE IN VOLLEYBALL PLAYERS}

\begin{abstract}
This paper sought to investigate the effect of different thermal stress situations (WBTG) on heart rate (FC), energy expenditure (GE), water loss (PH) and water intake (CA) in young volleyball players. For such, 09 young male volleyball players from Umuarama Volleyball participated of this research ( $16.5 \pm 0.9$ years). The variables FC, GE, PH and CA were measured during two training sessions $(\mathrm{S} 1, \mathrm{~S} 2)$ with similar volume $(85 \mathrm{~min})$ and intensity but, with different thermal stress situations (S1: WBTG: $22.62^{\circ} \mathrm{C}$ and $\mathrm{S} 2 \mathrm{WBGT}$ : $26.55^{\circ} \mathrm{C}$ ). The thermal stress (WBGT) in the training environment was determined by a globe thermometer model ITWTG2000. The FC and the GE were measured by a Suunto Team Pod. PH was calculated by the difference between the body weight at the beginning and at the end of the training sessions. The CA during the training sessions was "ad libitum" but the total volume ingested was calculated using $180-\mathrm{ml}$ plastic cups. For the data analysis, the paired T test (dependent samples) was used. The significance index adopted was $\mathrm{p}<0.05$. After the analysis of the data, similar FC (S1: $135.5 \pm 16.3 \mathrm{bpm}$ vs. S2: $141.5 \pm 21.5 \mathrm{bpm})$, GE (S1: $994.44 \pm 267.4 \mathrm{Kcal}$ vs. S2: $881.2 \pm 259.6$ Kcal), PH (S1: $0.6 \pm 0.2 \mathrm{Kg}$ vs. S2: $0.4 \pm 0.3 \mathrm{Kg}$ ) and CA (S1: $1120 \pm 345 \mathrm{ml}$ vs. S2: $760 \pm 429 \mathrm{ml}$ ) were observed in both training sessions. The results indicate that a little variation in the environmental thermal stress does not markedly affect the FC, GE, PH and CA during volleyball training sessions.
\end{abstract}

KEYWORDS: Thermal stress; Volleyball; Heart rate.

\section{Introdução}

O Brasil é conhecido como um país tropical, onde predominam temperaturas elevadas na maior parte do seu território. Essas condições climáticas nem sempre são favoráveis à prática de exercícios físicos ou esportes. Quando a temperatura ambiente encontra-se muito elevada, assim como a umidade relativa do ar, cria-se um verdadeiro desafio aos mecanismos de regulação da temperatura corporal que nem sempre conseguem equilibrar a produção e dissipação de calor, criando uma situação que pode agredir severamente a homeostase térmica do organismo (SILVERTHORN, 2010)

O exercício físico realizado em ambiente quente impõe uma sobrecarga adicional sobre os mecanismos termorregulatórios do organismo, independentemente do tipo de atividade e da idade do praticante (GOMES; CARNEIRO-JUNIOR; MARINS, 2013; SHIRREFFS et al., 2004; VIMIEIRO-GOMES; RODRIGUES, 2001). As reações químicas do metabolismo energético podem intensificar a pro-

DOI: https://doi.org/10.25110/arqsaude.v19i3.2015.5681

${ }^{1}$ Esp. em Fisiologia do Exercício - Professor Colaborador - Unipar /Departamento de Educação Física - alanpablo@unipar.br

${ }^{2}$ Acadêmico de Educação Física - PIC/Unipar - roni.ps@ @hotmail.com

${ }^{3}$ Acadêmico de Educação Física - PIC/Unipar - marcos.ant.16@hotmail.com

${ }^{4}$ Acadêmico de Educação Física - PIC/Unipar - jeffersonbagarolo@gmail.com

${ }^{5}$ Acadêmico de Educação Física - PIC/Unipar - vargas bru@hotmail.com

${ }^{6}$ Acadêmico de Educação Física - PIC/Unipar - augusto088@gmail.com

Endereço para correspondência: Rua Marialva, 5734 - Conjunto Residencial Ouro Verde II, Bloco B5, apto 22. Umuarama - Pr. CEP:87502-100. Telefone para contato: (44)91449500 
dução de calor em um nível muito superior à sua perda. Durante o exercício intenso, o metabolismo pode atingir níveis de 20 a 25 vezes acima dos valores de repouso em indivíduos treinados, chegando a aproximadamente $20 \mathrm{kCal} / \mathrm{min}$, o que pode aumentar a temperatura central em $1^{\circ} \mathrm{C}$ a cada 5 a 7 minutos (McARDLE; KATCH F; KATCH V, 2011).

A média da temperatura central normal oscila entre $36,5^{\circ} \mathrm{C}$ a $37^{\circ} \mathrm{C}$, podendo se elevar temporariamente para a faixa de $38,3{ }^{\circ} \mathrm{C}$ a $40^{\circ} \mathrm{C}$ durante o exercício intenso (HALL, 2011). Em situações nas quais o acúmulo de calor é excessivo, a ponto de agredir a homeostase térmica do organismo, mecanismos compensatórios são ativados com a finalidade de restabelecer o equilíbrio térmico e manter a integridade corporal. A estimulação das glândulas sudoríparas e a redução do tônus vasoconstritor da pele são os principais mecanismos ativados em resposta a um aumento súbito da temperatura central (POWER; HOWLEY, 2014).

Condições ambientais de calor e umidade elevados podem comprometer o desempenho no exercício de vários modos. Esses incluem, aumento na degradação de glicogênio muscular, acúmulo excessivo de lactato, maior produção de radicais livres, diminuição do fluxo sanguíneo muscular, desidratação e hipertermia (McARDLE; KATCH F; KATCH V, 2011; POWER; HOWLEY, 2014). Assim, esses fatores em conjunto podem contribuir para o desenvolvimento de fadiga muscular durante o exercício no calor, interferindo negativamente no metabolismo muscular, função cardiovascular, equilíbrio hídrico e funcionamento do sistema nervoso central (SILVERTHORN, 2010).

A exposição à temperaturas elevadas durante a realização de exercícios físicos não limita apenas o desempenho físico, mas também expõe o praticante ou atleta à doenças induzidas pelo calor (MARINS, 1998). Segundo Fox; Bowers; Foss (1991) esses distúrbios são assim classificados por ordem de gravidade progressiva: 1- câimbras induzidas pelo calor; 2 - síncope induzida pelo calor; 3 - exaustão térmica e 4 - intermação (insolação). A intermação poderá ocorrer quando a temperatura corporal se elevar até a variação crítica de $40,5^{\circ} \mathrm{C}$ a $42,2^{\circ} \mathrm{C}$. Nessa temperatura central, o indivíduo apresentará sintomas de desorientação, desconforto abdominal, vômitos e algumas vezes delírio com eventual perda de consciência (HALL, 2011).

É importante destacar que os mecanismos físicos envolvidos na perda de calor compreendem a convecção, condução, radiação e a evaporação (MARINS, 1998). Entre elas, a evaporação proporciona a principal defesa contra o superaquecimento (McARDLE; KATCH F; KATCH V, 2011). Os demais mecanismos, condução, irradiação e convecção, têm menor importância durante a prática de exercícios, principalmente os mais intensos e prolongados (CARVALHO; MARA, 2010).

A eficiência do resfriamento corporal pela evaporação da água do suor está diretamente relacionada a umidade relativa do ar, visto que, em ambientes secos a evaporação é mais rápida do que em ambientes úmidos (SILVERTHORN, 2010). Em média 0,58 Kcal de calor é perdido por cada grama de água que evapora (HALL, 2011), portanto para cada litro de suor evaporado da superfície do corpo, em torno de $580 \mathrm{kcal}$ de energia calorífica são eliminadas, o que se torna de fundamental importância durante a realização de exercícios físicos (MARINS, 1998).
Do ponto de vista fisiológico, o voleibol é caracterizado como um esforço físico intermitente, que exige dos jogadores deslocamentos rápidos em alta intensidade, seguidos por períodos mais calmos de baixa intensidade. Os esforços de alta e baixa intensidade intercalados durante toda a partida requerem que o jogador de voleibol tenha os sistemas de fornecimento de energia aeróbico e anaeróbico alático bem desenvolvidos (KOLEY; SINGH; SANDHU, 2010). É um esporte essencialmente praticado em ginásio fechado, porém exposto as adversidades atmosféricas como temperatura e umidade elevadas.

Uma partida ou sessão de treinamento de voleibol requer uma quantidade substancial de energia para sua realização, resultando em gasto energético significativo, com valores próximos a $650 \mathrm{Kcal} / \mathrm{h}$ (VIMIEIRO-GOMES; RODRIGUES, 2001). Como o fornecimento de energia pelas vias metabólicas apresentam um rendimento por volta de 34\% (McARDLE; KATCH F; KATCH V, 2011) com o restante sendo convertido em energia térmica (calor), a prática da presente modalidade resulta em grande produção de calor metabólico.

Ao longo das sessões de treinamento de voleibol ou mesmo durante as competições esportivas em ambiente quente, podem ocorrer graves prejuízos à integridade corporal do atleta, assim como, limitações ao seu desempenho se não forem tomadas as devidas precauções. O controle da ingestão adequada de líquido, o monitoramento do estresse térmico do ambiente de treino/jogo e a identificação de sinais ou sintomas relacionados à síncope ou exaustão pelo calor, são procedimentos de extrema importância para assegurar a saúde e maximizar o desempenho dos atletas (POWER; HOWLEY, 2014). Vale destacar que, a desidratação e a exaustão pelo calor são condições fisiológicas graves que ocorrem em consequência da exposição excessiva ao clima úmido e quente e que nem sempre são notadas imediatamente (GONZÁLEZ-ALONSO; CRANDALL; JOHNSON, 2008; McARDLE; KATCH F; KATCH V, 2011).

Portanto, devido à escassez de pesquisas sobre o tema e o interesse em compreender a dinâmica termorregulatória durante a prática esportiva do voleibol, a presente pesquisa teve como objetivo investigar o efeito de diferentes situações de estresse térmico (WBGT) sobre a frequência cardíaca (FC), gasto energético (GE), perda hídrica $(\mathrm{PH})$ e consumo de água (CA) em jovens atletas de voleibol, buscando fornecer subsídios para melhor elaboração de programas de treinamento físico em condições atmosféricas adversas, assim como assegurar a realização segura dos regimes de treinamento.

\section{Material e Método}

Participaram do estudo 09 indivíduos do sexo masculino (idade: $16,55 \pm 0,88$ anos), integrantes da equipe juvenil de voleibol do município de Umuarama, todos saudáveis e envolvidos com o treinamento de rendimento há pelo menos 1 ano. O estudo foi aprovado pelo Comitê de Ética em Pesquisa Envolvendo Seres Humanos da Universidade Paranaense - UNIPAR, sob Certificado de Apresentação para Apreciação Ética (CAAE) n. ${ }^{\circ}$ 20282513.5.0000.0109.

Inicialmente foi realizada a avaliação antropométrica dos participantes do estudo. Para a aferição do peso cor- 
poral foi utilizada uma balança com precisão de $0,05 \mathrm{Kg}$ da marca FILIZOLA ${ }^{\circledR}$ e para mensuração da estatura um estadiômetro com precisão de $1 \mathrm{~mm}\left(\right.$ CARDIOMED $\left.^{\circledR}\right)$.

Durante as duas sessões de treinamento foram mensurados: a FC, o CA e o peso corporal antes e após o treinamento para determinar a $\mathrm{PH}$. A FC além de ter sido utilizada como um indicador de intensidade do exercício, também foi usada para estimar o GE das sessões. Para a mensuração da FC foi utilizado o equipamento Suunto Team Pod (SUUN$\mathrm{TO}^{\circledR}$ ) que engloba um receptor e fitas de tórax. Todos os atletas foram monitorados simultaneamente e durante o mesmo espaço de tempo ( $85 \mathrm{~min}$ ). Os dados referentes a FC foram registrados digitalmente e analisados no software Suunto Team Manager $\left(\mathrm{SUUNTO}^{\circledR}\right)$ para determinar a FC média e o GE. O CA foi "ad libitum" sendo o volume total ingerido medido por meio de copos descartáveis de $180 \mathrm{ml}$ devidamente identificados com o nome dos atletas. Foi sugerido aos atletas que ingerissem o volume de água no copo em sua totalidade, visando facilitar a identificação do CA da sessão. Para a pesagem antes e após a sessão de treinamento, os atletas foram orientados a utilizar o mínimo possível de vestimentas, realizar a micção e se secarem antes da pesagem. Para tal finalidade foi utilizada uma balança com precisão de $0,05 \mathrm{Kg}$ da marca FILIZOLA ${ }^{\circledR}$. A PH foi calculada a partir da diferença entre o peso corporal no início e final da sessão.

Para avaliação do estresse térmico do ginásio foi utilizado um termômetro de globo da marca INSTRUTEMP ${ }^{\circledR}$ modelo ITWTG2000, devidamente calibrado. O instrumento também foi utilizado para coletar informações adicionais do ambiente, sendo elas: temperatura ambiente/ar (temperatura do bulbo seco), a temperatura do globo negro (medida da carga de calor radiante) e umidade relativa do ar (temperatura do bulbo úmido).

De acordo com as recomendações do Colégio Americano de Medicina do Esporte (ACSM, 1996; ACSM, 2007) referente à prevenção de doenças provocadas pelo calor durante treinamentos e competições, o padrão mínimo de monitoramento deve considerar o "índice de temperatura global de bulbo úmido" (WBGT: wet-bulb globe temperature) que é comporto pelas seguintes medidas: temperatura do bulbo seco $\left(\mathrm{T}_{\mathrm{bs}}\right)$, a temperatura do globo negro $\left(\mathrm{T}_{\mathrm{g}}\right)$ e a temperatura do bulbo úmido $\left(\mathrm{T}_{\mathrm{bu}}\right)$.

O índice WBGT é utilizado no meio esportivo e militar para avaliar o risco de exposição ao calor, sendo muito apropriado para esta finalidade, pois incorpora em sua medida as variáveis: carga de calor radiante (temperatura do globo negro - $\mathrm{T}_{\mathrm{g}}$ ), temperatura do ar (temperatura do bulbo seco - $\mathrm{T}_{\mathrm{bs}}$ ) e umidade relativa do ar (temperatura do bulbo úmido - $\mathrm{T}_{\mathrm{bu}}$ ). A equação para determinação do índice WBGT é a seguinte: $\mathrm{WBGT}=0,7 \mathrm{~T}_{\mathrm{bu}}+0,2 \mathrm{~T}_{\mathrm{g}}+0,1 \mathrm{~T}_{\mathrm{bs}}$.

$\mathrm{O}$ monitor de estresse térmico utilizado no estudo mensura a $\mathrm{T}_{b u}, \mathrm{~T}_{\mathrm{g}}$ e $\mathrm{T}_{\mathrm{bs}}$ e calcula automaticamente o índice WBGT expresso em ${ }^{\circ} \mathrm{C}$.

Os procedimentos descritos acima foram realizados em 2 sessões de treinamento com intervalo de 35 dias entre elas. Buscou-se realizar os procedimentos da pesquisa em dias que apresentassem uma diferença de aproximadamente $4^{\circ} \mathrm{C}$ no WBGT do ginásio, visando a avaliar os atletas sob diferentes situações de estresse térmico.

Os dados foram expressos em média e desvio padrão por meio da estatística descritiva. Para verificar a normalidade dos dados foi utilizado o teste de Shapiro-Wilk, confirmando a distribuição normal. Para identificar as diferenças nas variáveis $\mathrm{FC}, \mathrm{GE}, \mathrm{PH}$ e $\mathrm{CA}$ entre as 2 sessões de treinamento foi utilizado o teste $\mathrm{T}$ pareado (amostras dependentes). $\mathrm{O}$ índice de significância adotado foi de $\mathrm{p}<0,05$. O software utilizado foi o IBM ${ }^{\circledR}$ SPSS $^{\circledR}$ STATISTICS for Windows Versão 22.

\section{Resultados}

O tempo médio das sessões de treinamento foi de 85 minutos, excluindo-se o aquecimento e o alongamento que não foram monitorados.

O Quadro 1 descreve as características do ambiente e o índice WBGT do ginásio onde foram realizadas as 2 sessões de treinamento.

Quadro 1: Características do ambiente e índice WBGT nas 2 sessões de treinamento

\begin{tabular}{|l|c|c|c|c|}
\hline Sessão de treinamento & WBGT $\left({ }^{\mathbf{0}} \mathbf{C}\right)$ & TA $\left(\mathbf{T}_{\mathbf{b s}}\right)\left({ }^{\mathbf{0}} \mathbf{C}\right)$ & $\mathbf{T g}\left({ }^{\mathbf{0}} \mathbf{C}\right)$ & UR (Tbu) \\
\hline Sessão 1 (S1) & 22.62 & 29.77 & 29.55 & 39.82 \\
\hline Sessão 2 (S2) & 26.55 & 30.75 & 31.15 & 60.72 \\
\hline
\end{tabular}

WBTG: wet bulb globe temperature (temperatura global de bulbo úmido); TA: temperatura ambiente $\left(\mathrm{T}_{\mathrm{BS}}\right)$; TG: temperatura do globo negro (calor radiante); UR: umidade relativa do $\operatorname{ar}\left(\mathrm{T}_{\mathrm{bu}}\right)$. Equipamento: termômetro de globo INSTRUTEMP ${ }^{\circledR}$ modelo ITWTG2000.

A S2 apresentou valores de TA, $\mathrm{T}_{\mathrm{G}}$ e UR maiores que a $\mathrm{S} 1 . \mathrm{O}$ WBGT na $\mathrm{S} 2$ estava $3,93^{\circ} \mathrm{C}$ acima dos valores encontrados na $\mathrm{S} 1$. No quadro 1 é possível observar que a UR foi o principal fator influenciador do maior estresse térmico na $\mathrm{S} 2$.

A Tabela 1 expressa os valores de média e desvio padrão para a idade e medidas antropométricas dos atletas.

Tabela 1: Característica da amostra do estudo

\begin{tabular}{ccc}
\hline Idade (anos) & Peso Corporal (Kg) & Estatura (cm) \\
$16,55 \pm 0,88$ & $77,35 \pm 12,51$ & $184,44 \pm 8,48$ \\
\hline
\end{tabular}

A Tabela 2 expressa os dados referentes a variáveis
FC, GE, PH e CA coletados nas 2 sessões de treinamento com diferentes índices de estresse térmico.

\begin{tabular}{cccc} 
Tabela 2: FC, GE, PH e CA nas sessões de treinamento \\
\hline Variáveis analisadas & $\begin{array}{c}\text { Sessão } \mathbf{~} \\
\text { WBGT }=\mathbf{2 2 . 6 2}{ }^{\circ} \mathbf{C}\end{array}$ & $\begin{array}{c}\text { Sessão } \mathbf{2} \\
\text { WBGT }=\mathbf{2 6 . 5 5}{ }^{\circ} \mathbf{C}\end{array}$ & $\begin{array}{c}\text { Significância } \\
\text { (p) }\end{array}$ \\
FC (bpm) & $135,5 \pm 16,3$ & $141,5 \pm 21,5$ & \\
GE $(\mathrm{Kcal})$ & $994,4 \pm 267,4$ & $881,2 \pm 259,6$ & 0,423 \\
PH (Kg) & $0,60 \pm 0,20$ & $0,45 \pm 0,38$ & 0,120 \\
CA (mL) & $1120 \pm 345$ & $760 \pm 429$ & 0,233 \\
\hline
\end{tabular}

Valores médios e desvio padrão para as variáveis FC, GE, PH e CA das 2 sessões de treinamento. WBTG: wet bulb globe temperature (temperatura global de bulbo úmido).

Foi encontrado uma grande variação entre valores 
mínimos e máximos em todas as variáveis analisadas. Os valores mínimos e máximos para $\mathrm{S} 1$ e $\mathrm{S} 2$ respectivamente foram: FC (108-155bpm; 111-175bpm), GE (532-1374Kcal; $472-1191 \mathrm{Kcal}), \mathrm{PH}(0,40-0,90 \mathrm{Kg} ; 0,20-1,30 \mathrm{Kg})$ e CA $(540-$ $1620 \mathrm{~mL} ; 180-1620 \mathrm{~mL})$. Destaca-se também que em nenhuma das variáveis do estudo (FC, GE, PH, CA) foram encontradas diferenças significativas entre as duas sessões de treinamento com diferentes condições de estresse térmico.

\section{Discussão}

O voleibol é considerado um esporte com características metabólicas intermitentes e geralmente é praticado em ginásio fechado. Essas características somadas podem causar uma grande sobrecarga fisiológica, em especial, nos sistemas cardiorrespiratório e termorregulatório (KUNSTLINGER; LUDWIG; STEGEMANN, 1987).

A proposta principal da pesquisa foi investigar o efeito de diferentes situações de estresse térmico sobre a frequência cardíaca, gasto energético, perda hídrica e consumo de água em jovens atletas de voleibol. Como pode ser observado no quadro 1 , o índice WBGT em que se realizou os treinamentos foi de $22.62^{\circ} \mathrm{C}$ na $\mathrm{S} 1$ e $26,55^{\circ} \mathrm{C}$ na S2. A temperatura ambiente e a temperatura do globo negro foram semelhantes em ambas as sessões, porém, a umidade relativa do ar apresentou uma variação grande entre S1 (39.82\%) e S2 (60.72\%).

Power; Howley (2014) chamam a atenção para a importância da $\mathrm{T}_{\mathrm{bu}}$ (indicativo da umidade relativa do ar) na determinação do estresse térmico, quando se observa a equação para cálculo do índice de temperatura global de bulbo úmido $\left(\mathrm{WBGT}=0,7 \mathrm{~T}_{\mathrm{bu}}+0,2 \mathrm{~T}_{\mathrm{g}}+0,1 \mathrm{~T}_{\mathrm{bs}}\right.$ ). Pode-se observar que o $\mathrm{T}_{\mathrm{bu}}$ representa $70 \%$ do valor do índice, em comparação, o $\mathrm{T}_{\mathrm{bs}}$ representa apenas $10 \%$. As medições realizadas com o termômetro de globo no presente estudo confirmam que a umidade relativa do ar $\left(\mathrm{T}_{\mathrm{bu}}\right)$ foi a principal determinante do maior estresse térmico na S2 em comparação a S1.

De acordo com o posicionamento do Colégio Americano de Medicina do Esporte (ACSM, 2007), os riscos de acidentes causados pelo calor durante treinamentos e competições são classificados da seguinte forma: WBGT=10$-18,3^{\circ} \mathrm{C}$ : baixo risco de hipotermia ou hipertermia; $\mathrm{WBGT}=18,4-22,2^{\circ} \mathrm{C}$ : aumento no risco de doença por calor, pessoas com alto risco devem ser monitoradas ou não competir (não condicionados e não aclimatados); WBGT=22,3$-25,6^{\circ} \mathrm{C}$ : maior risco de hipertermia para todos (inclusive aclimatados e condicionados); WBGT $=25,7-27,8^{\circ} \mathrm{C}$ : perigo extremo de hipertermia para indivíduos não condicionados e não aclimatados e WBGT $>27,9^{\circ} \mathrm{C}$ : risco extremo de hipertermia, cancelar ou adiar evento ou treinamento.

No presente estudo observou-se que na $\mathrm{S} 1$, o risco de acidentes causados pelo calor foi muito baixo, porém, na $\mathrm{S} 2$ o risco foi elevado. Em qualquer valor de WBGT acima de $22,3^{\circ} \mathrm{C}$, é recomendado o monitoramento da ingestão líquido para prevenir acidentes causados pelo calor (ACSM, 1996, ACSM, 2007).

Em nenhuma das variáveis analisadas (FC, GE, PH e CA) foram encontradas diferenças significativas entre as duas sessões de treinamento com diferentes situações de estresse térmico, porém, alguns detalhes importantes puderam ser observados. Com relação a PH, constatou-se um estado inicial de desidratação, com redução próxima a $1 \%$ do peso corporal em grande parte dos atletas na S1, mesmo com o consumo de água sendo "ad libitum". A redução de $1 \%$ da massa corporal na forma de líquido compromete a termorregulação, se chegar entre 3 e 5\% causa aumento da FC e redução do débito cardíaco, atingindo 7\% em geral causa colapso durante o exercício (CARVALHO, MARA, 2010). Na S2 a $\mathrm{PH}$ foi menor, mesmo com a sessão apresentando WBGT mais elevado. Uma possível explicação para a menor PH na $\mathrm{S} 2$, mesmo não sendo significativa em relação a $\mathrm{S} 1$, seria a melhor aclimatação dos atletas ao ambiente quente, uma vez que houve um período de 35 dias entre S1 e S2, onde foram conduzidos vários treinamentos no calor. Power; Howley (2014) relatam que séries regulares de exercícios realizadas em condições ambientais de temperatura elevada resultam no rápido desenvolvimento de aclimatação ao calor, que promove adaptação máxima em torno de 14 dias. Essa aclimatação pode resultar na diminuição da temperatura central corporal e possivelmente minimizar PH por transpiração.

O CA no presente estudo esteve próximo do valor relatado por Vimiero-Gomes; Rodrigues (2001) no qual, em uma sessão de treinamento de voleibol, foi ingerido em média 0,9 litros de água. Entretanto, observou-se uma grande variação individual nesse consumo, o que pode ser constatado pelo elevado desvio padrão desta variável (TABELA 2). O CA entre os atletas variou de 540 a $1620 \mathrm{~mL}$ na S1 e de 180 a $1620 \mathrm{~mL}$ na S2. Essa grande variação individual também pode ser observada na $\mathrm{PH}$, em que a variação foi de 0,40 $0,90 \mathrm{Kg}$ na $\mathrm{S} 1$ e $0,20-1,30 \mathrm{Kg}$ na S2. Possivelmente, houve uma influência da maior umidade relativa do ar observado na S2 em relação a S1 sobre essas variáveis. Outro fator que pode ter influenciado essa grande variação individual no CA e na PH entre os atletas, seria a função específica de cada jogador em quadra. Considerando que alguns atletas se deslocam e saltam mais do que outros, não é surpresa encontrar grandes variações individuais em virtude dos diferentes níveis de esforço físico realizado.

Com relação a $\mathrm{FC}$, foi observado uma similaridade nas duas sessões, demonstrando que realmente a intensidade em ambas foi semelhante e que a variação no estresse térmico não foi suficiente para causar ajustes mais profundos nos parâmetros cardiovasculares. O GE foi estimado por software, utilizando a FC média como parâmetro. Esse procedimento envolve um erro considerável, porém, foi utilizado buscando-se ter uma visão geral do consumo energético durante o treinamento. O GE não apresentou variações significativas entre as sessões. Vale ressaltar que para uma estimativa mais precisa do gasto energético seria necessário equipamento de alto custo e difícil manuseio, por este motivo não foi utilizado na presente pesquisa, sendo essa uma das limitações do estudo.

Frente a esses resultados, fica claro que a diferença do índice WBGT entre as duas sessões de treinamento de voleibol analisadas nesta pesquisa se mostrou muito pequena para induzir ajustes fisiológicos significativos. Acreditou-se, inicialmente, que um pequeno estresse térmico adicional (aproximadamente 3 a $4^{\circ} \mathrm{C}$ a mais no índice WBGT) seria suficiente para elevar a frequência cardíaca de exercício e estimular os indivíduos a ingerirem maior quantidade de líquido devido a desidratação. A hipótese de aumento da FC foi levantada devido ao grande número de evidências sobre 
o efeito da temperatura sobre os parâmetros cardiovasculares em exercício (POWER; HOWLEY, 2014; McARDLE; KATCH F; KATCH V, 2011; GONZÁLEZ-ALONSO; CRANDALL; JOHNSON, 2008) porém, aparentemente existe a necessidade de um estresse térmico mais elevado para se observar esses ajustes.

\section{Conclusão}

Uma pequena variação $\left(3,93^{\circ} \mathrm{C}\right)$ no índice de estresse térmico entre duas sessões de treinamento de voleibol não foi capaz de induzir alterações significativas nas variáveis FC, GE, PH e CA.

Finalizando, observou-se que quase todos os atletas apresentaram algum nível de desidratação nas duas sessões de treinamento, indicando a necessidade de controle mais rigoroso sobre os intervalos para hidratação e, principalmente, na quantidade de água ingerida, visando minimizar as consequências deletérias do treinamento realizado em situações de estresse térmico elevado.

Por conseguinte, existe a necessidade de mais estudos considerando uma maior faixa de variação de WBGT a fim de determinar seus reais efeitos sobre os parâmetros cardiovasculares, a ingestão de água, o gasto energético e a perda hídrica durante a prática do voleibol. É importante destacar também que pesquisas envolvendo um maior número de participantes poderia facilitar a identificação de possíveis modificações nessas variáveis frente aos diferentes índices de WBGT.

\section{Referências}

\section{AMERICAN COLLEGE OF SPORTS MEDICINE}

POSITION STAND. Exertional heat illness during training and competition. Med. Sci. Sports Exerc. v. 39, n. 3, p. $556-572,2007$

\section{AMERICAN COLLEGE OF SPORTS MEDICINE}

POSITION STAND. Heat and cold illnesses during distance running. Med. Sci. Sports Exerc. v.28, n.12, p.i-x 1996.

CARVALHO, T.; MARA, L. S. Hidratação e Nutrição no Esporte. Rev. Bras. Med. Esporte. v.16, n.2, p.144-148, 2010.

FOX, E. L.; BOWERS, R. W.; FOSS, M. L. Bases

Fisiológicas da Educação Física e dos Desportos. 4ed. Rio de Janeiro: Guanabara Koogan, 1991. 518p.

GONZÁLEZ-ALONSO, J.; CRANDALL, C. G.; JOHNSON, J. M. The cardiovascular challenge of exercising in the heat. J. Physiol. v.586, n. 1, p.45-53, 2008.

GOMES, L. S.; CARNEIRO-JUNIOR, M. A.; MARINS, J. C. B. Respostas termorregulatórias de crianças no exercício em ambiente de calor. Rev. Paul. Pediatr. v. 31, n.1, p.104110, 2013.

HALL, J. E. Tratado de Fisiologia Médica. 12. ed. Rio de Janeiro: Elsevier, 2011. 1151p.
KOLEY, S.; SINGH, J.; SANDHU, J. S. Anthopometric and physiological characteristics on Indian inter-university volleyball players. Journal of Human Sport \& Exercise. v.5, n.3, p389-399, 2010.

KUNSTLINGER, U.; LUDWIG, H. G.; STEGEMANN, J. Metabolic Changes During Volleyball Matches. Int. J. Sports. Med. v.8, n. 5 p.315-322, 1987.

MARINS, J. C. B. Mecanismos físicos de perda de calor e fatores associados relacionados ao exercício. R. Min. Educ. Fís. Viçosa, v. 6, n.2, p. 5-20, 1998.

MARINS J. C. B. Exercício físico e calor - Implicações fisiológicas e procedimentos de hidratação. Revista

Brasileira de Atividade Física e Saúde. v. 1, n.3, p. 26-38, 1996.

McARDLE, W. D.; KATCH, F. I.; KATCH, V. L. Fisiologia do Exercício: Nutrição, Energia e Desempenho Humano. 7. ed. Rio de Janeiro: Guanabara Koogan, 2011. 1061p.

POWER, S.K.; HOWLEY, E. T. Fisiologia do Exercício: teoria e aplicação ao condicionamento e ao desempenho. 8ed. Barueri - São Paulo, 2014. 650p.

SHIRREFFS, S. M.; ARAGON-VARGAS, L. F.; CHAMORRO, M.; MAUGHAN, R. J.; SERRATOSA, L.; ZACHWIEJA, J. J. The Sweating Response of Elite Professional Soccer Players do Training in the Heat. Int. J. Sports Med. v. 26, p90-95, 2004.

SILVERTHORN, D. U. Fisiologia Humana: uma abordagem integrada. 5. ed. Porto Alegre: Artmed, 2010. 992p.

VIMIEIRO-GOMES, A.C.; RODRIGUES, L. O. C. Avaliação do estado de hidratação dos atletas, estresse térmico do ambiente e custo calórico do exercício durante sessões de treinamento em voleibol de alto nível. Rev. Paul. Educ. Fís., São Paulo, v.15, n.2, p.201-211, jul/dez. 2001.

Recebido: 28/05/2015 Aceito: 12/11/2015 